\title{
Web-Based Tool Integration: A Web Augmentation Approach
}

\author{
Oscar Díaz ${ }^{1}$, Josune De Sosa ${ }^{2}$, Cristóbal Arellano ${ }^{1}$, and Salvador Trujillo ${ }^{2}$ \\ 1 ONEKIN Group, University of the Basque Country (UPV/EHU), Spain \\ \{oscar.diaz, cristobal.arellano\}@ehu.es \\ 2 IKERLAN Research Centre, Mondragon, Spain \\ \{jdesosa, strujillo\}@ikerlan.es
}

\begin{abstract}
Desktop tools are steadily being turned into web applications. Tool integration then becomes a question of website integration. This work uses Web Augmentation techniques for this purpose. An integration layer is deployed on top of the existing Web-based tools that augments the rendering of those tools for the integration experience. Layers are specified through a statechart-like DSL and transformed into JavaScript.
\end{abstract}

\section{Introduction}

Tool integration is a matter of reducing "accidental complexity" due to the different semantics brought by each tool. Tools might differ on the data format, user-interface conventions, use of common functions, the process flow, etc [3]. Tool integration can be achieved on three different levels: the data source level, the business logic level, and the user interface (UI) level 4. UI integration has two significant benefits: (i) existing applications' UIs can be reused, and (ii) users already familiar with existing UIs do not have to learn how to work with new ones. So far, UI integration has been investigated at the component level where a bright new integration application is constructed from existing components [1]. However, tools are not components but full-fledged web applications. Portlets can fit this scenario but they impose a heavy footprint on both the tool provider and the tool consumer (i.e. the tool integrator) [2. This certainly hinders the openness and self-serviceness of the solution.

This work's research question is whether the use of Web Augmentation (WA) techniques can provide an alternative balance between expressiveness and selfserviceness. Rather than sophisticated and expressive solutions such as those of portlets or UI components, WA introduces a lightweight solution based on the front-end. WA is to the Web what Augmented Reality is to the physical world: layering relevant content/layout/navigation over the existing Web to customize the user experience. This is achieved through JavaScript using browser weavers (e.g. Greasemonkey). However, WA is hindered by being programming intensive and prone to malware. As a result, we resort to Domain-Specific Languages (DSLs) as a way to abstract away from the implementation details, ease user participation, and promote openness. We introduce CORSET, a DSL for Webbased tool integration based on process flows. 


\section{The Running Example}

The tools to be integrated include: Jazz Rational Team Concert 1 (hereafter, just $J a z z$ ) to be used for the management of the software development lifecycle, and LucidChart2, a tool for model design. Figure 1(a) outlines the process flow between Jazz and LucidChart. First, the user opens Jazz to check the workload. Task 24 has been assigned: "design the $x m l$ schema". This task involves the design of a UML class diagram. This requires to move to a different tool: LucidChart. Once created, the UML diagram is assigned a permalink. This permalink is to be shared with the rest of the Jazz community. To this end, the user goes back to task 24 in Jazz. Finally, the user copy\&paste the permalink as an artefact associated to the Jazz task. In this scenario, the integration functionality is in the user's head: no support is given to sustain neither the control flow (e.g. when to navigate from Jazz to LucidChart, and vice versa) nor the data flow (e.g. the diagram permalink that flows between the websites). This is the very purpose of CORSET. Figure1(b) introduces a CORSET layer to sustain the

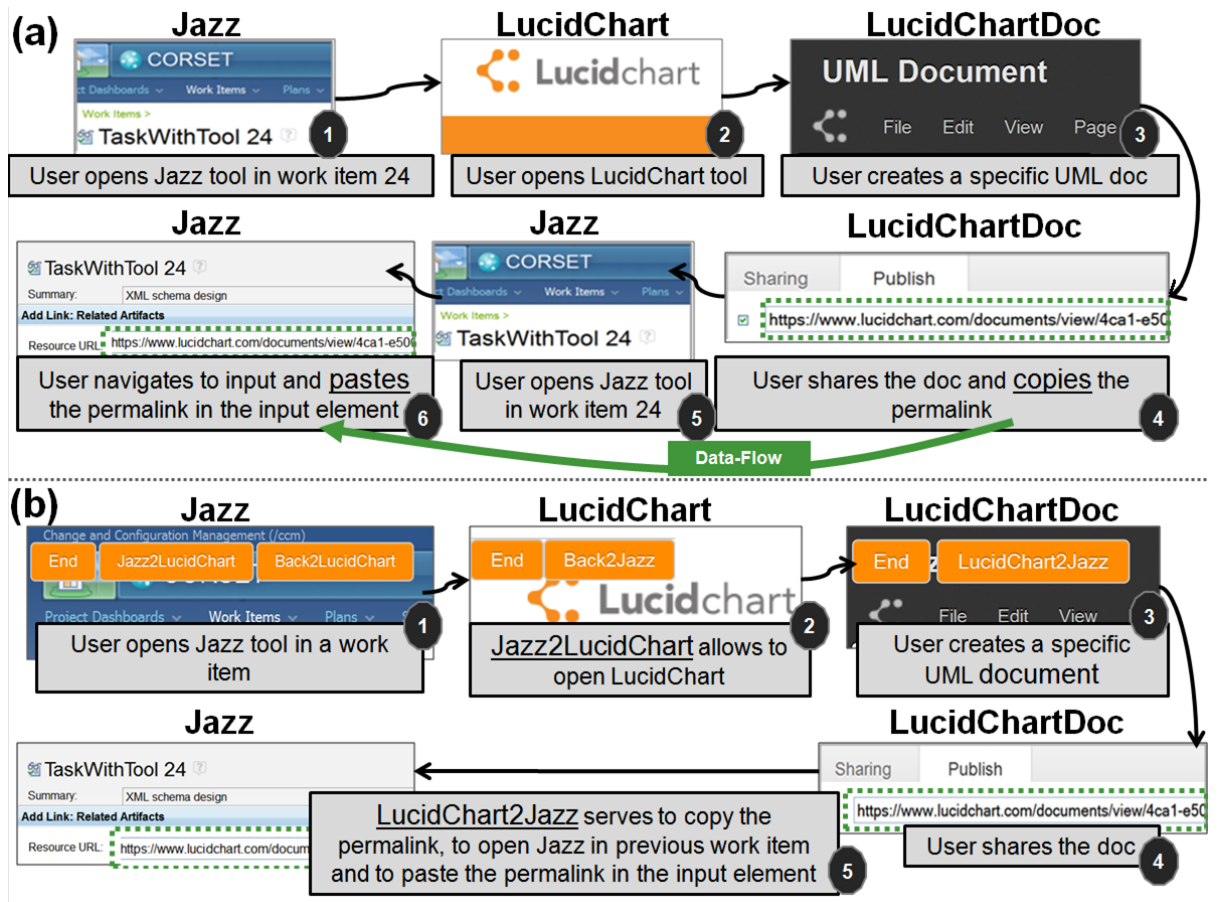

Fig. 1. Integrating the websites of Jazz and LucidChart. (a) The process expands along the two websites. (b) A CORSET layer is interspersed to support this integration.

\footnotetext{
${ }^{1}$ https://jazz.net/projects/rational-team-concert/

2 http://www.lucidchart.com/
} 
sample scenario. The Jazz website is augmented with three additional buttons: Jazz2LucidChart, Back2LucidChart and End. The former serves to seamless navigate to LucidChart the very first time (creating a bright new UML diagram) whereas Back2LucidChart handles posterior visits. Likewise, the LucidChart website now exhibits two new buttons. Back2Jazz serves to navigate back to the departing state at Jazz. Unlike the previous case, this navigation is contextual in the sense that navigation is parameterized by the permalink of the current LucidChart artefact. It is worth noticing that at any moment the user can move away from these two tools, and browses other web applications. At any moment, users can finalize the process by clicking the End button.

\section{CORSET}

CORSET uses statecharts to describe the integration scenario. Figure 2 shows the CORSET expression for the running scenario. This diagram is transformed into a JavaScript program. A process-based UI-centric approach to tool integration entails a control flow, a data flow and the UI augmentations.

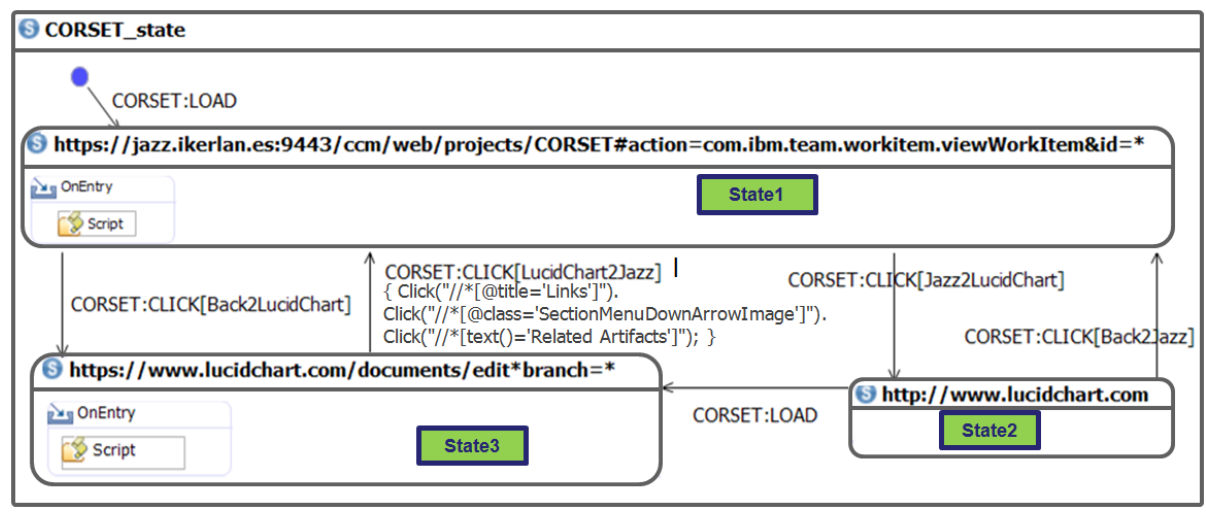

Fig. 2. CORSET expression for the running scenario

Control Flow. It is captured through statecharts: a state is characterized by a URL pattern that identifies the set of pages that participate in accomplishing a given task; a transition normally implies moving between websites. For our running example, we have three simple states, one for Jazz (https://jazz.ikerlan.es:9443/ccm/web/projects/CORSET... see Figure 2) and two for LucidChart (https://www.lucidchart.com/documents/edit... and www.lucidchart.com). A transition has an event, a condition and a target state. Events denote abstractions of happenings which are meaningful for the integration purpose. They are signalled by CORSET and abstracted from lowlevel DOM events. So far, two event types are considered: CLICK, that denotes pushing a CORSET button, and LOAD, that is an abstraction of the DOM load 
event. This event is risen by CORSET when a tool loads an UI that matches a state of the CORSET at hand. Finally, an action denotes a CORSET Script. These scripts describe actions to be enacted in the target tool as a result of this transition. For instance, moving from State 3 in LucidChart to State1 in Jazz requires the previous "internal navigation" of three clicks till the right UI is reached (see Figure 2 where the "Click" action mimics user clicks)

Dataflow. Some transitions might be turned into contextual links, i.e. links that carry data from the source to the target. Broadly, we have to mimic copy\&paste as conducted by the user. Hence, CORSET offers a high-level "copy" and "paste" operation, and uses state variables as the clipboard. This is part of the CORSET script (not shown in the figure). For instance, the script Copy("XPath expression").Into(\$stateVariable) keeps in the state variable the output of evaluating the XPath.

UI Integration Augmentation. A CORSET expression also has a rendering counterpart: the buttons. Buttons are automatically generated from transitions. Button placement is based on the assumption that the place that holds some data of interests (the data being extracted) coincides with the place where the control flow should be governed. If no such data exists, buttons are inserted in the upper left of the window. This heuristic permits the CORSET engine to automatically generate the UI.

\section{Conclusions}

We investigate Web Augmentation techniques for tool integration. By using a DSL, we strive to shelter users from JavaScript and describe the integration declaratively as statecharts. A must follow-on is to conduct usability studies among the tool users to assess whether CORSET expressiveness fulfils their requirements.

\section{References}

1. Daniel, F., Soi, S., Tranquillini, S., Casati, F., Heng, C., Yan, L.: From People to Services to UI: Distributed Orchestration of User Interfaces. In: Hull, R., Mendling, J., Tai, S. (eds.) BPM 2010. LNCS, vol. 6336, pp. 310-326. Springer, Heidelberg (2010)

2. Díaz, O., Rodriguez, J.: Portlets as Web Components: an Introduction. Journal of Universal Computer Sciences (JUCS) 10(4), 454-472 (2004)

3. Thomas, I., Nejmeh, B.: Definitions of Tool Integration for Environments. IEEE Software 9(2), 29-35 (1992), http://dx.doi.org/10.1109/52.120599

4. Westermann, U., Jain, R.: Toward a Common Event Model for Multimedia Applications. IEEE MultiMedia 14(1), 19-29 (2007) 